\title{
A new mix together algorithm for wireless network state
}

\author{
Moyi Duan XiangZhong Wang \\ Software College \\ Nanjing railway vocational and technical college \\ Nanjing, China \\ e-mail:duanmoyi@163.com
}

\begin{abstract}
This paper propose a new fusion method (Signal Fusion based on signal Wavelet transform and Date Association, SFWD), its purpose is to performance issues by the signal generated by the influence of interference resolution process in the wireless network transmission. Firstly, based on wavelet transform reduces the long correlation signal, and using Kalman filtering and data association based fusion algorithm. At the same time, the relationship between signal and interference factors were studied by comparing the experimental simulation, the results show that the method has a certain degree of adaptability, the error between the fusion results with the original signal is 7.13 .
\end{abstract}

Keywords: interference signal; fusion; long correlation; wavelet; Data Association

\section{Introduction}

With the rapid development of wireless networks, interference signal between the more and more serious. At the same time as the Mac layer of the competition and network congestion and other factors, which have an important change on the signal state will. How to restore the original signal at the receiving end by noise interference has become the focus of current research. At the same time, the study found that in recent years the actual signal has self similarity and long-range correlation characteristics of [1,2], distribution and Poisson previously thought has great difference. In this regard, the domestic scholars have done a lot of work. The literature [3] sent by artificial noise way in multiple antenna systems, through secret by means of multiple relay nodes, which can be well applied in cooperative relay networks. The literature [4] presented a distributed algorithm for cooperative interference mechanism, and the optimal jamming weights by binary search method. The literature [5] through mobile control node event loss rate is restricted to a certain range, the bound event loss probability. Document [6] in a comprehensive analysis of the existing problems of traditional link measurement method based on the average RSSI (Received Signal, Strength Indication) and the mean CCI (Commodity Channel Index) measurement method. The literature on change characteristics of [7] link time, found great changes occurred in link quality between nodes in wireless sensor networks, resulting in data transmission between nodes is not stable. The literature [8] the link quality evaluation method does not take into account the effect of incomplete packet on the forward link, a evaluation model under non perceived packet loss and link quality, and will link packet wireless sensor network loss is divided into perceived packet loss and no loss of perceptual component. The literature [9] for data compression and weighted fairness

problem is seldom considered in wireless sensor network congestion control method of existing, proposes an epsilon approximation and weighted fairness of congestion control algorithm. The literature [10], slotted CSMA/CA of saturated[11] in the case of (Carrier Sense Multiple Access with Collision Avoidance) algorithms for modeling. Document [12] non-persistent CSMA backoff based on a relatively simple and effective model. Through the discussion of the literature [13] congestion degree and channel busy ratio of network rate. According to the literature [14] hidden nodes and carrier sense limits proposed Ad hoc network optimal throughput quantification analysis method. Document $[15,16]$ study found that congestion not only by the node physical ability and node data flow, and data and the interference area flow.

On the basis of the above work, this paper puts forward a new fusion method (Signal Fusion based on signal Wavelet transform and Date Association, SFWD). The method using the wavelet transform to reduce the long correlation signal, and Kalman filter and associated data fusion based on signal, at the same time, the simulation experiment of in-depth analysis of the original signal state changes in the interference signal influence, results show that the method is effective.

\section{The model is introduced}

Assume the existence of a wireless network channel model as shown in figure 1. All nodes in a wireless ad hoc network, synchronization with Mac protocol effectively. Each node has only one antenna, working in half duplex mode, and the structure and function of the identical node. If in a certain period of time the node $\mathrm{S}$ as the signal 
transmitting end node $(\mathrm{i}=1,2, \mathrm{Mi}, \ldots, \mathrm{n})$ as the relay terminal, node $\mathrm{R}$ as the signal receiving end, node $\mathrm{N}$ as a relay node interference noise source (no interference to nodes $\mathrm{S}$ and $\mathrm{R}$ ). The relay node to obtain the noisy signal from a transmitting end and interference source in the same time slot, and the signal is transmitted to the receiving end.

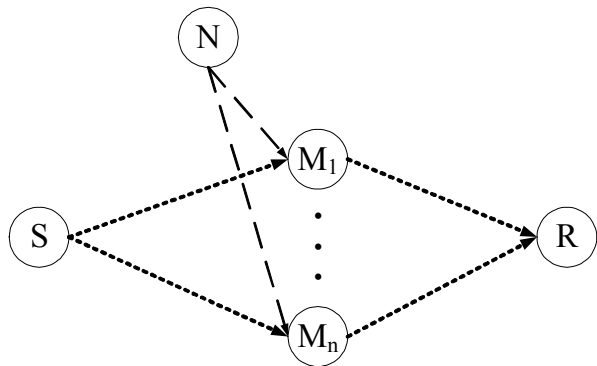

Figure 1 wireless channel model

Make a time slot $\mathrm{t} \mathrm{S}$ sends the same signal are transmitted to the relay nodes $\mathrm{Mi}$, the effective signal and noise signal interference sources of $\mathrm{Mi}$ will be transmitted to the receiving end with R. Signal state assumption that nodes send $\mathrm{S}$ to $\mathrm{Y}(\mathrm{T})=[\mathrm{y} 1$, $\mathrm{y} 2, \ldots, \mathrm{yk}], \mathrm{K} \geq 1$, YK may be the capacity, power, transmission rate. Due to the different interference node $\mathrm{N}$ and each relay node Mi distance, interference effects generated will be different, then the node $\mathrm{R}$ to accept the signal to the relay node Mi will vary. The node $\mathrm{N}$ interference signal on $\mathrm{Mi}(\mathrm{T})=[\varepsilon 1, \varepsilon 2, \ldots \mathrm{Yi}, \varepsilon \mathrm{n}]$, the signal is received by $\mathrm{Mi}(\mathrm{T})$ can be expressed as:

$$
Y_{i}(t)=\sqrt{P_{i S}} Y(t) h_{i S}+\sqrt{P_{i N}} \varepsilon_{i} h_{i N}+e
$$

Among them, PiS is the transmitted signal power nodes $\mathrm{S}$ and Mi, hiS is the channel between $\mathrm{S}$ and Mi, PiN for sending the interference power node $\mathrm{N}$ to $\mathrm{Mi}, \varepsilon$ I $\mathrm{N}$ interference to signal $\mathrm{Mi}$, e as the channel of Gauss white noise.

In order to be able to restore the original signal node $\mathrm{S}$ sends in the node $\mathrm{R}$, the signals of each relay node Mi sends the data fusion. But the study found that the self similarity with the actual signal, if the direct signal on the receive node in the $\mathrm{R}$ fusion, may produce large error, so this wavelet transform is adopted to reduce the long correlation characteristics of the signal, and then the data association for data fusion, in order to restore the original signal.

Multiresolution analysis is often used in wavelet transform, the original signal according to the layer decomposition to the corresponding space. Mallat algorithm [17,18] is a multi resolution analysis, by constructing two filters, for frequency division of the sample:

$\left\{\begin{array}{l}A_{j+1}=H A_{j} \\ D_{j+1}=G A_{j}\end{array}\right.$

Among them, $\mathrm{H}$ low pass filter, $\mathrm{G}$ high pass filter, $\mathrm{J}$ decomposition level, $\mathrm{j}=1,2, \ldots$.

Typically, sample data that need more the number of decomposition is more, at the same time, if the sample stability is poor, so the number of decomposition will be more. The current research the wavelet basis on which data, and decomposed into many hierarchical optimal performance has not been substantive results, so can consider to use multiple wavelet base flow to compare the treatment effect. $\mathrm{Aj}$ and $\mathrm{Dj}$ are known as the approximation coefficients and wavelet coefficients in the resolution of $2-\mathrm{j}$.

\section{The algorithm design}

3.1 The described design of SFDF algorithm

Assuming as shown in Figure 1 Wireless self organization network model, each node in half duplex mode, and the structure and function of the identical node. In a certain period of time the node $\mathrm{S}$ as the signal transmitting end node $(\mathrm{i}=1,2, \mathrm{Mi}, \ldots, \mathrm{n})$ as the relay terminal, node $\mathrm{R}$ as the signal receiving end, node $\mathrm{N}$ as a relay node interference noise source (no interference to nodes $\mathrm{S}$ and $\mathrm{R}$ ). The relay node to obtain the noisy signal from a transmitting end and interference source in the same time slot, and the signal is transmitted to the receiving end.

Make a time slot $\mathrm{t}$ node $\mathrm{S}$ will be the same signals are transmitted to the relay nodes $\mathrm{Mi}$, the effective signal and noise signal interference sources of Mi will be transmitted to the receiving end with $\mathrm{R}$. Signal state assumption that nodes send $\mathrm{S}$ to $\mathrm{Y}(\mathrm{T})=[\mathrm{y} 1, \mathrm{y} 2, \ldots, \mathrm{yk}], \mathrm{K} \geq 1$, YK may be the capacity, power, transmission rate. Due to the different interference node $\mathrm{N}$ and each relay node $\mathrm{Mi}$ distance, interference effects generated will be different, then the node $\mathrm{R}$ to accept the signal to the relay node Mi will vary. The node $\mathrm{N}$ interference signal on $\mathrm{Mi}(\mathrm{T})=$ $[\varepsilon 1, \varepsilon 2, \ldots, \varepsilon \mathrm{n}]$, PiS as the transmitted signal power nodes $\mathrm{S}$ and Mi, hiS is the channel between $\mathrm{S}$ and Mi, PiN for sending the interference power node $\mathrm{N}$ to $\mathrm{Mi}, \varepsilon \mathrm{I} \mathrm{N}$ interference to signal $\mathrm{Mi}$, e as the channel of Gauss white noise. 
2222 The design of SFDF algorithm

Step 1 in a time slot $\mathrm{t}$ initialization node $\mathrm{S}$ sends a signal state $\mathrm{Y}(\mathrm{T})$ and node $\mathrm{N}$ interference signal $(\mathrm{t})$;

Step 2 by formula (4) calculation of relay node Mi receives signal Yi ( $\mathrm{t}$ );

$Y_{i}(t)=\sqrt{P_{i S}} Y(t) h_{i S}+\sqrt{P_{i N}} \varepsilon_{i} h_{i N}+e$

Step 3 is a step ahead prediction data Yi ( $\mathrm{t} \mid \mathrm{t}-1)$ and type (5) signal obtained new information V (T);

$V(t)=Y_{i}(t \mid t-1)-Y_{i}(t)$

Step 4 according to equation (6) signal on the receiving end of the update:

$Y_{i}(t \mid t)=Y_{i}(t \mid t-1)+V(t)+X(t)$

Among them, $\mathrm{X}(\mathrm{T})$ FARIMA (P, D, q) information model for long signal update, is calculated as follows:

(I) using (7) choosing a truncation parameter $\mathrm{M}$ and parameter $\mathrm{N}$ :

$X(n)=\sum_{m=0}^{M-1} c(m) Z_{\alpha}(n-m)$

Among them, $\mathrm{n}=0,1, \ldots, \mathrm{N}-1$;

(II) by the formula (8) calculation of $\mathrm{C}(\mathrm{m}), \mathrm{m}=0,1, \ldots, \mathrm{M}+\mathrm{N}-1$, and the base two FFT algorithm of $\mathrm{C}(\mathrm{m})$ of the discrete Fu Liye transform $(\mathrm{m})=\mathrm{DM}+\mathrm{N}(\mathrm{C}(\mathrm{m}))$;

$\left\{\begin{array}{l}c(0)=1 \\ c(m)=\frac{\Gamma(m+d)}{\Gamma(d) \Gamma(m+1)}\end{array}\right.$

Among them, gamma gamma function;

(III) to generate the standard symmetric alpha stable distribution variable $\mathrm{Z} \mathrm{M}+\mathrm{N}$ independent and identically distributed (m), and then use FFT algorithm to calculate the base two period extended $\mathrm{Z}(\mathrm{m})$ of the discrete Fu Liye transform $(\mathrm{m})=\mathrm{DM}+\mathrm{N}(\mathrm{Z}(\mathrm{m}))$;

(IV) using base two FFT algorithm to calculate the product () the inverse Fu Liye transform, and

$$
X(t)=D_{M+N}^{-1}(\hat{c} \hat{Z}(m))
$$

Among them,;

Step 5 type (10) parameters of R signal fusion nodes receive, obtain the original signal Y (T) estimator [19,20];

$\hat{Y}(t \mid t)=\sum_{i=1}^{n} Y_{i}(t \mid t) \beta_{i}(t)$

Among them, $\beta$ I (T) is the integration of parameters;

Step 6 combined (11) to predict the next time slot state:

$Y(t+1 \mid t)=F(t+1) \hat{Y}(t \mid t)+r(t+1)$

Among them, $\mathrm{R}(\mathrm{t}+1)$ for drift, $\mathrm{F}(\mathrm{t}+1)$ as the state vector;

Step $7 \mathrm{t}=\mathrm{t}+1$, jump to Step 2, until the end of the cycle;

step 8 The end of algorithm.

3.2 The design of SFWD algorithm 
Firstly, based on wavelet transform reduces the long correlation signal, and using Kalman filtering and data association based fusion algorithm.

Step 1 in a time slot $\mathrm{t}$ initialization node $\mathrm{S}$ sends a signal state $\mathrm{Y}(\mathrm{T})$ and node $\mathrm{N}$ interference signal $(\mathrm{t})$;

Step 2 according to equation (1) calculation of relay node Mi receives signal Yi (t);

Step 3 according to the signal of long-range dependence and self similarity characteristics, the type (2) signal Yi wavelet transform is shown for node $\mathrm{R}$ received $(\mathrm{T})$ decomposition, to get the approximate coefficient $\mathrm{Aj}$ and wavelet coefficient Dj;

Step 4 is approximate coefficient $\mathrm{Aj}$ and wavelet coefficient $\mathrm{Dj}$ (3), the inverse wavelet transform reconstruction shown reduced long-range dependence of signal Yi $(\mathrm{t})$ :

$$
A_{j-1}=H^{*} A_{j}+G^{*} D_{j}
$$

Among them, $\mathrm{H}^{*}$ and $\mathrm{G}^{*}$ are dual operator $\mathrm{H}$ and $\mathrm{G}$;

Step 5 based on the predicted data of $\mathrm{Yi}(\mathrm{t} \mid \mathrm{t}-1)$, the new information is obtained by signal:

$$
V(t)=Y_{i}(t \mid t-1)-Y_{i}(t)
$$

Step 6 according to the new information, signal receiving updates:

$$
Y_{i}(t \mid t)=Y_{i}(t \mid t-1)+V(t)+K(t)
$$

Among them, K (T) as Kalman gain;

Step 7 node of R signal parameter fusion, obtain the original signal Y (T) estimator [21-23]:

$$
\hat{Y}(t \mid t)=\sum_{i=1}^{n} Y_{i}(t \mid t) \beta_{i}(t)
$$

Among them, $\beta \mathrm{I}(\mathrm{T})$ is the integration of parameters;

Step 8 according to type (5) and Kalman gain, to predict the next time slot state:

$$
Y(t+1 \mid t)=F(t+1) \hat{Y}(t \mid t)+r(t+1)
$$

Among them, $\mathrm{R}(\mathrm{t}+1)$ for drift, $\mathrm{F}(\mathrm{t}+1)$ as the state vector;

Step $9 \mathrm{t}=\mathrm{t}+1$, jump to Step 2, until the end of the cycle;

Step 10 The end of algorithm.

The algorithm uses Kalman filter, its time complexity is $\mathrm{O}(\mathrm{N} 3)$, the time complexity of the algorithm can be expressed as $\mathrm{O}(\mathrm{Mn} 3)$.

\section{4. simulation experiments}

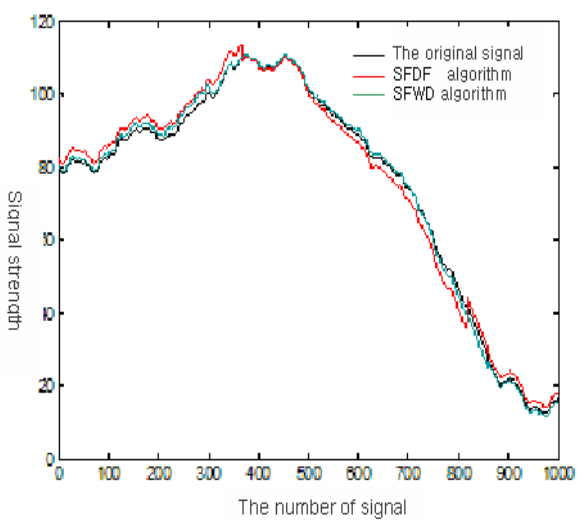

Fig. 2 Comparison of fusion algorithm
In order to verify the effectiveness of the proposed methods, first set up network topology shown in Figure 1 in OPENT. The rate $\mathrm{S}$ signals season point is $2279 \mathrm{~kb} / \mathrm{sec}$, the fractal Brown motion (Fractional Brownian Motion, FBM) model has 1000 long signal SFBM. The correlation coefficient $\mathrm{H}=0.9$. Figure 3 shows the fusion results of SFDF algorithm and SFWD algorithm for SFBM signal. As you can see from Figure 3, the signal SFBM is more closer to the convergence of SFWD algorithm, two algorithms of the standard deviation as shown in table 1.At the same time, the signal state $\mathrm{Y}(\mathrm{T})$ in which a component capacity $\mathrm{YK}$ analysis. According to the description of the literature[24-26], the SNR is $\gamma$ I to node $\mathrm{Mi}$, channel 
transmission bandwidth is $\mathrm{W}$, the capacity of $\mathrm{YK}$ can be expressed as:

$$
\begin{aligned}
& y_{k}=\frac{W}{2} \log \left(1+\frac{P_{S}\left|h_{i S}\right|^{2}\left|h_{i R}\right|^{2} \lambda^{2}}{\sigma_{e}^{2} \lambda^{2}}\right) \\
& -\frac{W}{2} \log \left(1+\frac{\left|h_{i S}\right|^{2}}{\left|h_{i N}\right|^{2}+\sigma_{e}^{2}}\right)
\end{aligned}
$$

Among them, $\sigma$ E2 white noise power, lambda for the amplification factor.

Due to the very important influence interference interference between the nodes $\mathrm{N}$ and Mi relay nodes distance $\mathrm{L}$ of the original signal[274-29], this paper studies the relation between capacity and interference distance is L, as shown in figure 2. As you can see from Figure 2, the overall tendency with increasing interference distance L, capacity is increased, and tends to be stable. But in the interference distance is small, the capacity curve degree is obvious. At the same time, it can be found that the SFWD algorithm, capacity curve depicts the closer to the original traffic, its performance is better than SFDF algorithm.

Table 1 fusion algorithm of standard deviation

\begin{tabular}{ll}
\hline Algorithm & standard deviation \\
\hline SFDF & 6.53 \\
SFWD & 6.25
\end{tabular}

Fig. 3 capacity and interference distance L

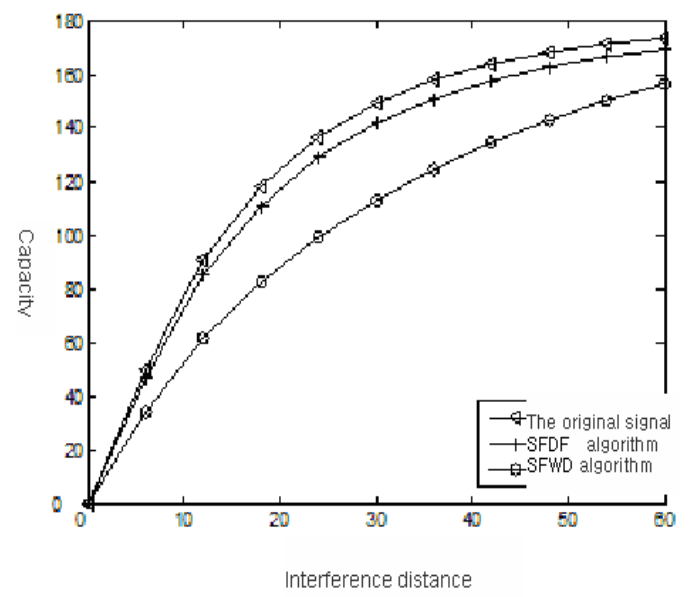

Through the above research can be seen, SFWD algorithm based on SFDF algorithm improved is more suitable for the description of wireless network, and its performance is more consistent with the actual situation.

\section{Conclusion}

According to the performance by the signal generated by the influence of interference in the wireless network transmission process, this paper based on the SFDF algorithm presents a new signal state fusion algorithm of SFWD. The algorithm combines FARIMA model and data fusion of signal, and the simulation experiment of SFDF algorithm and SFWD algorithm performance, and analyzes the changes between capacity and interference distance, the results show that the SFWD algorithm has more adaptability. In the follow-up study, can consider to establish the performance of the wireless network transmission mechanism with the physical layer security evaluation system.

2013 VNI forecast the key content of the new report is forecast for IPv6 traffic, although new not assigned IPv4 address space in technology have dried up, but the IPv6 flow is still relatively small. However, IPv6 traffic is growing[30].

"The most likely scenario is, at the end of this year, IP flow rate of 3.1\% will be IPv6, and by the end of last year was only $1.3 \%$," Sumits said, "by the end of 2017, IP traffic will be 23.9 is IPv6."

In 2012 to the end of 2017, IPv6 traffic will grow more than 50 times.

In view of the present these predictions, the biggest problem is that the modern network can be appropriately extended scale to fit the predicted growth. For this problem, Cisco hope service provider can give a positive answer. 
Sumits said: "we believe that the service provider has the ability to meet the growing demand, they can in the traffic management in the use of intelligent network and content distribution technology, to ease the traffic pressure."

Network traffic prediction and analysis is the basis of network management, because of its affected by many factors, is not smooth, nonlinear changes the traditional forecasting methods, forecasting accuracy is low, therefore, put forward a network traffic prediction model of wavelet decomposition and SVM combination. The simulation results show that, compared with other forecasting models, the algorithm can improve the network traffic prediction accuracy, is a model for forecasting strong generalization ability, good robustness of network traffic.

\section{Acknowledgment}

The National Science Education "Twelfth Five-Year Plan" project of Ministry of education stage of research (FJB110092), computer key project of Ministry of education of Higher Vocational College (jzw590107z09), National Natural Science Foundation of China (61128005 - 03), Jiangsu province college adult education research "research" in 2009, the adult higher education stage study results

\section{Reference}

[1] Leland W. E., Taqqu M. S., Willinger W., et al. On the self-similar nature of Ethernet traffic (extended version)[J]. IEEE/ACM Transactions on Networking, 1994, 2(1): 1-15.

[2] Lazarou G. Y., Baca Julie, Frostv S., et al. Describing network traffic using the index of variability[J]. IEEE /ACM Transactions on Networking, 2009, 17 (5): 1672-1683.

[3] S. Goel, R. Negi. Guaranteeing secrecy using artificial noise[J]. IEEE Transactions on Wireless Communication, 2008, 7(6): 21802189.

[4] G. Zheng, L. Choo, K. Wong. Optimal cooperative jamming to enhance physical layer security using relays[J]. IEEE Transactions on Signal Processing, 2011, 59(3): 1317-1322.

[5] Bisnikm, Abouzeidaa, Isleraa. Stochastic event capture using mobile sensors subject to a quality metric[J]. IEEE Trans on Robotics, 2007, 23(4): 676-692.

[6] Sun Peigang, Zhao Hai, Luo Dingding, et al. The link quality measurement for wireless sensor network [J]. Journal of communication, 2007, 28 ( 10 ): 14-22.

[7] D. Couto, D. Aguayo, B. A. Chambers, et al. Performance of multihop wireless networks: shortest path is not enough[J]. ACM SIGCOMM Computer Communication Review, 2003, 33(1): 83-88.

[8] Shu Jian, Liu Linlan, Fan Youlei, Li Jun. Model [J]. Journal of China Institute of communications, evaluating wireless sensor network link quality under non perceived packet loss 2011, 32 (4): 103-111.

[9] Li Guohua, Li Jianzhong, Gao Hong. Epsilon approximation and weighted fairness of congestion control algorithm for wireless sensor network [J]. Chinese Journal of computers, 2011, 34 (11): 2197-2210.

[10] Yang L., Dawson C. W., Brown M. R., et al. Neural network and GA approaches for dwelling fire occurrence prediction. Knowledge-Based Systems, 2006, 19(4): 213-219.

[11] dragon picture, Sun Zhengshun, Li Chunwen, Jiang Peigang, Liu Jinhua. The multifractal wavelet model . Chinese Journal of computers, a new network traffic 2004, 27 (8): 1074-1082.

[12] cluster lock, Han Liangxiu, Liu Yan, Gao Chuanshan. The discrete wavelet transform of network traffic multifractal model based on . Journal of communication, 2003, 24 (5): 43-48.

[13] Lin Jipeng, Liu Junhua. Wavelet support vector machine algorithm research . Journal of Xi'an Jiao Tong University, based on 2005, 39 (8): 816-819

[14] Yang Jinfang, Zhai Yongjie, Wang Dongfeng, Xu Daping. Prediction of time series of . support vector regression based on Chinese Journal of mechanical engineering, 2005, 25 (17): 110-114.

[15] Wang Dingcheng, Fang Tingjian, Gao Lifu, Ma Yongjun. The support vector machine regression on-line modeling and application of . control and decision, 2003, 18 (1): 89-91.

[16] Lin Y. K.. Reliability of a computer network in case capacity weight varying with arcs, nodes and types of commodity. Reliability Engineering and System Safety, 2007, 92 (5): 646-652.

[17] Gunawan I.. Redundant paths and reliability bounds in gamma networks. Applied Mathematical Modeling, 2008,32 (4): $588-594$.

[18] Xiaolong Wu, Shahram Latifi. Substar reliability analysis in star networks. Information Sciences, 2008,178 (10): $2337-2348$.

[19] Felemban E., Lee C. G., Ekici E.. MMSPEED: multi-path multi-SPEED protocol for QoS guarantee of reliability and timeliness in wireless sensor networks. IEEE Trans. Mobile Computing, 2006, 5 (6): 738-754.

[20] Tan Xianhai, Huang Yuanhui, Jin Weidong. Random Early Detection Algorithm of Self-sim ilar Traffic. Journal of Southwest Jiaotong University, 2008, 43 (1): 19-24.[Xianhai Tan, Huang Yuanhui, Jin Weidong. The self-similar flow random early detection method . Jour nal of Southwest Jiao Tong University, 2008, 43 (1): 19-24. 